\title{
Perfil epidemiológico dos acidentes com animais peçonhentos na região norte do Brasil, estado do Amazonas/AM
}

Os acidentes por animais peçonhentos apontam um problema de saúde pública que causam morbimortalidade e o conhecimento desse agravo por meio da epidemiologia é importante por subsidiar informações para a criação de estratégias que ajudem na solução dos acidentes. Trata-se de um estudo retrospectivo, descritivo, de caráter epidemiológico, registrados de 2018 a 2019, incluído o tipo de animal envolvido no acidente. Foram notificados 3.078 casos de acidentes, sendo no ano de 2018, 22 casos e em 2019, 3.056 casos notificados, 13 munícipios registraram esses acidentes, nos anos de 2018 e 2019 , a faixa etária que mais se acidentou foi a de 20-39 anos, população essa está envolvida em atividades econômicas locais e, portanto, expostas aos animais peçonhentos, o sexo masculino também é mais acometido pelos acidentes com animais peçonhentos, sendo no ano de 2019 , os maiores números de casos, homens com 2.236 de casos notificados enquanto que mulheres 820 casos registrados. Devido ao fato do homem, está ativamente em trabalho de pesca, bem no período de atividade econômica, eles estão mais susceptíveis aos acidentes, cabendo haver uma educação em saúde, na medida de prevenção, da população do estado do Amazonas.

\section{Epidemiological profile of accidents with venomous animals in the northern region of Brazil, state of Amazonas/AM}

Accidents by venomous animals point to a public health problem that causes morbidity and mortality, and the knowledge of this disease through epidemiology is important because it subsidizes information for the creation of strategies that help in the solution of accidents. This is a retrospective, descriptive, epidemiological study, registered from 2018 to 2019, including the type of animal involved in the accident. 3,078 accident cases were reported, with 22 cases in 2018 and 3,056 reported cases in 2019, 13 municipalities recorded these accidents, in the years 2018 and 2019, the age group that most suffered accidents was $20-39$ years, this population is involved in local economic activities and, therefore, exposed to venomous animals, the male gender is also more affected by accidents with venomous animals, with the highest number of cases in 2019 , men with 2,236 cases reported while than women 820 registered cases. Due to the fact that men are actively engaged in fishing, right in the period of economic activity, they are more susceptible to accidents, and there is a need for health education, as far as prevention is concerned, for the population of the state of Amazonas.

Keywords: Amazonas; Accident; Venomous; Public health; Animals.

\section{Topic: Toxicologia}

Reviewed anonymously in the process of blind peer
Received: 01/05/2020

Approved: 29/06/2020
Adriano Menino de Macedo Júnior

Centro Universitário Natalense, Brasil

http://lattes.cnpq.br/4134152465913204

http://orcid.org/0000-0001-6367-1088

adrianomenino2016@gmail.com
Referencing this:

MACEDO JUNIOR, A. M.. Perfil epidemiológico dos acidentes com animais peçonhentos na região norte do Brasil, estado do Amazonas/AM. Nature and Conservation, v.13, n.3, p.24-31, 2020. DOI: http://doi.org/10.6008/CBPC2318-2881.2020.003.0003 


\section{INTRODUÇÃO}

Animais peçonhentos são aqueles que possuem a capacidade de produzir toxinas líquidas, a partir de glândulas específicas. A injeção de tais substâncias toxicas e letais, é feita em presas ou em predadores, através de dentes ocos, ferrões, nematocistos e pelos, como autodefesa ou também na atividade de caça de suas presas. Essas toxinas eficientes podem alterar a fisiologia de diferentes sistemas, gerando sequelas incapacitantes temporárias ou definitivas, podendo levar a óbito quando não adequadamente manejados (DIAS JUNIOR, 2018).

Os acidentes com animais peçonhentos são considerados um problema de saúde pública, em todo o mundo, principalmente em países com regiões tropicais e subtropicais, e no Brasil os principais animais que causam estes acidentes e são: serpentes, de escorpiões, de aranhas, de lepidópteros (mariposas e suas larvas), de himenópteros (abelhas, formigas e vespas), de coleópteros (besouros), de quilópodes (lacraias), de peixes, de cnidários (águas-vivas e caravelas), entre outros (ASSIS et al., 2019).

No Brasil, os acidentes ocasionados por aranhas, escorpiões e serpentes, tem sido a maior causa de intoxicações notificadas e na Região Norte do País, a maior taxa de notificações tem sido registrada por serpentes peçonhentas (SOUZA et al., 2019). A seriedade do assunto tem contribuído para os altos índices de morbidade e mortalidade, sobretudo nas zonas rurais, onde temos pessoas expostas a esses animais e com acesso a serviços de saúde limitados e escasso (MARTINS et al., 2018).

O Amazonas é um dos estados que integra a região norte do Brasil, e é caracterizado por ser a mais extensa das unidades federativas do país em área territorial, com 1.559.161,682 km2, o que chama atenção nessa para estar Região é seu perfil de natureza, coberta de reservas de flora, fauna e águas (SOUZA et al., 2019). As serpentes correspondem ao grupo de répteis Squamata com maior riqueza em florestas Neotropicais. Na América Latina, acidentes com serpentes constituem um relevante problema de saúde pública, causando altas taxas de morbidade e mortalidade. As principais serpentes de interesse médico nas Américas são da família Viperidae, subfamília Crotalinae (cascavéis, jararacas e surucucus), que possuem dentição solenóglifa, com dentes anteriores maiores e móveis, altamente especializados para a injeção de peçonha (OLIVEIRA et al., 2017).

Aranhas do gênero Loxosceles, comumente conhecidas como aranhas reclusas marrons, podem causar acidentes graves em humanos. Seu veneno tem uma poderosa ação proteolítica e hemolítica. A cada ano essas aranhas são a causa de um grande número de araneismo no Brasil. Essas aranhas são típicas na Região Norte, no estado do Amazonas (ALMEIDA et al., 2017).

Segundo a Organização mundial de Saúde (OMS), O Ministério da Saúde brasileiro por meio da portaria $\mathrm{n}^{\circ} 2.472$ de 31 de agosto 2010 inseriu os acidentes com animais peçonhentos como parte da Lista de agravos de Notificação Compulsória em território nacional devido ao alto número de casos previamente notificados por meio do SINAN, tornando este tema como de fundamental importância epidemiológica para o Brasil.

Os acidentes com animais peçonhentos, foram também classificados, pela Organização Mundial da 
Saúde, como uma doença tropical negligenciada devido ao fato de que estudos prévios observaram um risco maior desse tipo de agravo para pacientes com renda familiar mais baixa e em países com Índice de Desenvolvimento Humano é inferior, demonstrando por meio disso também a importância do atual estudo envolvendo a população regional (OMS, 2019).

As populações rurais são as principais vítimas desses acidentes, considerando a regularidade, a amplitude das ocorrências, a assistência e a dificuldade de acesso aos serviços de saúde nessas regiões (SOUZA et al., 2019). A etiologia desse problema pode ser advinda das transfigurações ocorridas no ambiente pelo qual o homem atua. Em virtude disso, as ações geradas pelo homem como o desmatamento florestal, por exemplo, promovem alterações no habitat tornando-o não mais favorável e peculiar para esses animais, promovendo assim uma relação íntima e contínua entre os animais e seres humanos (SALVI et al., 2018).

O Brasil dispõe atualmente dos seguintes sistemas de notificação: Sistema de Informação de agravos de Notificação (SINAN), Sistema Nacional de Informações Tóxico-Farmacológica (SINITOX/FIOCRUZ), Sistema de Informações Hospitalares do Sistema Único de Saúde/MS e o Sistema de Informação sobre Mortalidade (SIM), para obtenção de casos com animais peçonhentos (SILVA et al., 2018).

Mesmo com esse aparato conspirando a favor, a saúde pública não consegue controlar os acidentes ocasionados por animais peçonhentos, podendo ser pela dificuldade no acesso a unidades de saúde, contudo a população mais envolvida nesses acidentes são as que estão em maior contato com a zona rural do Amazonas, bem como a zona urbana do estado que também sofre com a precariedade sistema de saúde (ASSIS et al., 2019).

O presente estudo tem como objetivo relatar os casos de acidentes com animais peçonhentos no estado do Amazonas - AM, bem como a importância da notificação por parte da equipe de atendimento das unidades de saúde, assim é possível ter um maior conhecimento dos acidentes que mais acometem a Região Norte, e possivelmente trabalhar com a estratégia de distribuição de soro e melhor preparação do profissional com os casos das principais espécies causadoras dos acidentes em pessoas, aqui explanados.

\section{MATERIAIS E MÉTODOS}

Está pesquisa foi desenvolvida na forma descritiva e retrospectiva, de natureza epidemiológica, com dados fornecido e extraídos da base de dados do Sistema Nacional de Agravos de Notificação (SINAN), estatísticas essas disponibilizadas pelo DATASUS. Os dados foram filtrados na plataforma pelos anos de 2018 e 2019, para os casos notificados de acidentes com animais peçonhentos, no estado do Amazonas - AM, localizado na região Norte do Brasil - BR, as variáveis avaliadas na discussão foram ano de notificação em que aconteceu o acidente, tipo de animal peçonhento responsável pelo acidente, microrregiões IBGE de residência segundo o ano da notificação e a faixa Etária das pessoas que se acidentaram com os animais peçonhentos.

A metodologia da presente pesquisa utilizou também pesquisas bibliográficas, como revisão de literatura, a qual foi lastreada em artigos constantes na base eletrônica SciELO, Google acadêmico, Pubmed, MEDLINE (National Library of Medicine, USA - NLM), assim como repositórios de universidades públicas e 
privadas, empregando-se as palavras chave: Animais Peçonhentos, Saúde pública, perfil epidemiológico, Amazonas.

Todos os dados epidemiológicos coletados para análise foram selecionados e obtidos por meio do aplicativo TABNET, a partir de suas caixas de opções (linha, coluna e conteúdo). Por se tratar de dados secundários de um banco de domínio público, não foi necessário submeter o trabalho ao Comitê de Ética em Pesquisa em Seres Humanos (CEP).

\section{RESULTADOS E DISCUSSÃO}

Animais peçonhentos são aqueles que produzem venenos mortais e têm condições naturais para injetá-la em presas ou predadores. Essa condição é dada naturalmente por meio de dentes modificados, aguilhão, ferrão, quelíceras, cerdas urticantes, nematocistos entre outros. Esses animais são responsáveis por alta taxa de acidentes e mortalidades no estado do Amazonas - AM (SOARES et al., 2019).

Neste artigo podemos evidenciar o quantitativo e frequência anual, dos acidentes ocorridos em 2018 e 2019, no estado do Amazonas - AM. A tabela 1, a seguir demostra que foram notificados 0,71\% (22 casos) de acidentes com animais peçonhentos no não de 2018, contrariamente do ano de 2019 , foram registrados $99,28 \%$ ( 3.056 casos) de acidentes com estes animais.

Tabela 1: Notificações de acidentes com animais peçonhentos segundo o Ano.

\begin{tabular}{lll}
\hline Ano de notificação & Total & \\
\hline 2018 & 22 & \\
2019 & 3.056 & 3.078 \\
\hline Total & & \\
\hline
\end{tabular}

Um estudo realizado epidemiológico e descritivo, realizado no Brasil - BR, no período de 2007 e 2017, relatou que nesse período houve 95.215, notificações de acidentes de trabalho com animais peçonhentos, em trabalhadores vulneráveis, a pesquisa também ressaltou que no ano de 2007, foram notificados 7.830 casos e em 2017 foram registrados, 10.825 casos de acidentes causados por animais peçonhentos (SOUZA et al., 2019).

Os principais animais considerados de importância médica são as aranhas dos gêneros Phoneutria (Armadeira), Latrodectus (Viúva Negra) e Loxosceles (Aranha Marrom); as serpentes dos gêneros Crotalus (cascavel), Micrurus (coral verdadeira), Bothrops (jararaca) e Lachesis (surucucu); as lagartas do gênero Lonomia e os escorpiões do gênero Tityus. Dentro desse contexto, ainda devem ser mencionadas as abelhas africanizadas, que surgiram através do cruzamento acidental entre abelhas africanas e europeias no interior de São Paulo, em 1956. As abelhas, embora muito produtivas, apresentam riscos a pessoas, visto que possuem temperamento muito violento (VIEIRA et al., 2018). A tabela número 2, ilustram os acidentes notificados segundo a variável do tipo de acidente, segundo ano do registro.

De acordo com um estudo transversal descritivo, retrospectivo, clínico e epidemiológico, realizado na Região Norte do Brasil - BR, no período de 2012 a 2015, constatou vários acidentes por parte dos principais animais peçonhentos, existentes na Região, dentre eles destacou-se: 58.432 casos de acidentes por animais peçonhentos, durante o período analisado, sendo que a maioria causada por, serpentes 
(34.801 casos), seguido por escorpiões (13.040 casos) e aranhas (3.109 casos) (LOPES et al., 2017).

Tabela 2: Notificação de tipo de acidente segundo o Ano do acidente.

\begin{tabular}{llll}
\hline Tipo de acidente & 2018 & 2019 & Total \\
\hline Ignorado/branco & - & 12 & 12 \\
Serpente & 21 & 2.056 & 2.077 \\
Aranha & - & 196 & 196 \\
Escorpião & 1 & 492 & 493 \\
Lagarta & - & 25 & 25 \\
Abelha & - & 52 & 52 \\
Outros & - & 223 & 223 \\
\hline Total & 22 & 3.056 & 3.078 \\
\hline
\end{tabular}

Segundo Pinto et al. (2019), em seu estudo epidemiológico e transversal retrospectivo e descritivo, no período de 2014 a 2016, observou que as notificações um total de 430 casos notificados, onde, os maiores de acidentes com animais peçonhentos foram, ocasionados por botrópicos com 33\% ( $n=142)$, escorpião $49 \%$ $(n=211)$. A tabela número 3 , explana o total de casos onde ocorrem os acidentes com animais peçonhentos, segundo a variável, notificações por microrregião IBGE de residência de acordo com os anos de 2018 e 2019.

Tabela 3: Notificações por microrregião IBGE de residência de acordo com os anos.

\begin{tabular}{llll}
\hline Região de ocorrência & 2018 & 2019 & Total \\
\hline Rio Negro - AM & 3 & 256 & 259 \\
Japurá & 1 & 22 & 23 \\
Alto Solimões & 7 & 333 & 340 \\
Juruá & 5 & 163 & 168 \\
Tefé & - & 197 & 197 \\
Coari & 1 & 180 & 181 \\
Manaus & 2 & 396 & 398 \\
Rio Pedro da Eva & - & 131 & 131 \\
Itacoatiara & 1 & 345 & 346 \\
Parintins & - & 479 & 479 \\
Boca do Acre & 1 & 50 & 51 \\
Purus & - & 87 & 87 \\
Madeira & 1 & 417 & 418 \\
\hline Total & 22 & 3.056 & 3.078
\end{tabular}

Pode-se verificar que o maior número de casos prevaleceu em 2019, os munícipios mais acometidos foram os de Manaus ( $n=396)$, Madeira ( $n=417$ ) e Parintins ( $n=479)$. De acordo com Silva et al. (2019a), o estudo epidemiológico quantitativo, descritivo e retrospectivo, realizado no estado do Rio de janeiro, aponta em seus resultados 462 notificações no período de janeiro de 2007 a agosto de 2017, no qual a maior número de casos foi registrado na zona rural, com $42 \%$ de notificações.

Os acidentes nestas regiões estão intimamente relacionados a inundações, no Amazonas elas são graduais e conhecidas como enchentes ou cheias, afetando extensivamente os munícipios da Região. No período de 2015 a 2019, a população Ribeirinha que vive as margens dos rios, sofreu bastante com o impacto do desastre (SOUZA et al., 2017). Esses desastres graduais ocorrem em espaço-tempo distinto, no primeiro trimestre do ano, às enchentes atinge os municípios das calhas dos rios Juruá, Purus e Madeira, enquanto que no segundo trimestre, ocorre nos municípios das calhas do Solimões, Amazonas e Negro (FERREIRA BISNETO et al., 2019).

Esse tipo de desastre que ocorre no Amazonas acarreta consigo doenças e agravos ocasionados por microrganismos que contaminam a água, interferem no sistema de abastecimento de água e o principal de 
todos que é o foco do deste trabalho, trazem inúmeros animais peçonhentos, causando diversos acidentes (SOUZA et al., 2017).

Consoante, Organização Mundial de Saúde, afirmam que os acidentes em zonas rurais são mais comuns de ocorrer, é nesse contexto que corrobora com a literatura nacional, mas também com os estudos internacionais já que a própria Organização Mundial da Saúde, declarou os acidentes com animais peçonhentos uma Doença Negligenciada pelo fato de que acomete mais a população rural, de baixa renda e baixa escolaridade e que, portanto, possui menor acesso a um serviço de saúde de qualidade. Os acidentes com animais peçonhentos atingem todas as faixas etárias, na tabela número 4 , consta os casos notificados e suas respectivas idades.

Tabela 4: Notificação anual segunda a faixa etária.

\begin{tabular}{llll}
\hline Faixa Etária & 2018 & 2019 & Total \\
\hline Ignorado/branco & - & 1 & 1 \\
$<1$ Ano & - & 24 & 24 \\
$1-4$ & - & 83 & 83 \\
$5-9$ & 3 & 169 & 172 \\
$10-14$ & 6 & 301 & 307 \\
$15-19$ & 2 & 349 & 351 \\
$20-39$ & 6 & 1.136 & 1.142 \\
$40-59$ & 4 & 723 & 727 \\
$60-64$ & - & 103 & 103 \\
$65-69$ & 1 & 71 & 72 \\
$70-79$ & - & 75 & 75 \\
$\geq 80$ & - & 21 & 21 \\
\hline Total & 22 & 3.056 & 3.078 \\
\hline
\end{tabular}

Nesta pesquisa podemos verificar que a população mais acometida é da faixa etária de 20 a 39 anos de idade, as faixas posteriores a essa também demonstram serem bastante acometida. Em um estudo transversal, descritivo e retrospectivo, realizado na Região Serrana do Rio de Janeiro - RJ, no período de 2007 a 2015, teve maior registro de notificações na faixa etária de 20 a 59 anos, representando $70,27 \%$ ( $n=934$ casos) dos casos registrados, o estudo também mostra que a população da terceira idade, especificamente maior e igual 80 anos é a menos acometida pelos acidentes com animais peçonhentos (VIEIRA et al., 2018). Segundo os dados coletados nesta pesquisa, os homens apresentaram maior taxa de acidente por animais peçonhentos do que mulheres, como ilustrado na tabela número 5.

Tabela 5: Notificação anual segundo o sexo do indivíduo.

\begin{tabular}{llll}
\hline Ano de notificação & 2018 & 2019 & Total \\
\hline Sexo masculino & 17 & 2.236 & 2.253 \\
Sexo Feminino & 5 & 820 & 825 \\
\hline Total & 22 & 3.056 & 3.078 \\
\hline
\end{tabular}

A maior taxa de registros, ocorreu em homens, apresentando em 2018, com o percentual de 0,55\% ( $n=17$ casos) e em 2019, 72,6\% ( $n=2.236$ casos), diferente do sexo oposto que ocorreu em 2018 0,16\% ( $n=5$ casos) e em 2019 26,6\% ( $n=820$ ). O estudo que corrobora com os dados supracitados, foi o realizado na mesorregião do baixo Amazonas no estado do Pará entre o período de 2010 a 2016, onde o sexo masculino notificou $73,7 \%$ de casos e o sexo feminino apresentou 37,2\% de casos confirmados (SILVA et al., 2019b).

Outro estudo realizado por Vitória (2019), identificou-se que a maior parte das notificações, 
acometeram pessoas do sexo masculino (56,55\%) e o sexo feminino apresentou (43,43\%). Mas um estudo que aponta em seus resultados para a variável explanada na tabela 5, é o de Cunha et al. (2019), em seu estudo observacional, descritivo, analítico e retrospectivo, realizado no estado do Piauí, no período de janeiro 2007 até dezembro 2011, observou que do sexo mais acometido por acidentes com animais peçonhentos, os homens tiveram $82,4 \%(n=103)$ e mulheres com $17,6 \%(n=22)$ dos casos notificados.

\section{CONCLUSÕES}

Os acidentes ocasionados por animais peçonhentos são um problema de saúde pública que afeta as mais diversas localidades e esferas sociais, do estado do Amazonas. A frequência dos acidentes por esses tipos de animais na Região Norte é elevada se comparada com outras pesquisas realizadas voltadas a essa temática. No decorrer da construção do artigo, as análises de dados do estudo apontam, que os maiores acometidos são os indivíduos do sexo masculino, pescadores e pessoas em idade economicamente ativa, isso porque a base econômica do estado do Amazonas, principalmente da zona rural são atividades como a pesca e agricultura.

O acesso limitado de pessoas ao atendimento de saúde, tem elevado as taxas de mortalidade e morbidade local, por animais peçonhentos, a importância da notificação dos acidentes nas bases de dados e fundamento, para que as autoridades públicas tomem as devidas precauções e medidas. A criação de programas voltados à área de saúde, ações de educação em saúde de como pode prevenir esses acidentes, devem ser criadas e adotadas, criação de unidades de saúde, em maior territorialidade devem ser feitas com o objetivo de reduzir à mortalidade, uma vez que muitos desses animais, tem o veneno letal, e aumentar a expectativa de vida dessas pessoas.

\section{REFERÊNCIAS}

ALMEIDA, M. Q.; SALVATIERRA, L.; CARVALHO, T. G.; PRESTES, F. J.; BRESCOVIT, A. D.; GASNIER, T. R.. Long time not seen: Expanding the records of Loxosceles amazonica (Araneae: Sicariidae) in the Amazonas state, Brazil. Acta Amazônica, v.47, n.2, p.163-166, 2017

ASSIS, S. N. S.; LIMA, R. A.; RODRIGUES, J. J. P. Levantamento de acidentes com animais peçonhentos registrados em Tabatinga/AM, Brasil. Revista Gestão \& Sustentabilidade Ambiental, v.8, n.1, p.582-599, 2019.

CUNHA, V. P.; SANTOS, R. V. S. G.; RIBEIRO, E. E. A.; MAIA FILHO, A. L. M.; MARQUES, R. B.. Perfil epidemiológico de acidentes com animais peçonhentos no Piauí. 2019.

DIAS JUNIOR, F. D. A. C.. Acidentes por animais peçonhentos: caracterização epidemiológica no estado de Santa Catarina no período de 2014 a 2016. Monografia (Bacharelado) - Universidade do Sul de Santa Catarina, Tubarão, 2018

FERREIRA BISNETO, P.; ALCÂNTARA, J. A.; SILVA, I. M., SACHETT, J. D. A. G.; BERNARDE, P. S.; MONTEIRO, W. M.; KAEFER, I. L.. Coral snake bites in Brazilian Amazonia: perpetrating species, epidemiology and clinical aspects. Toxicon, v.175, p.7-18, 2019. DOI:

http://doi.org/10.1016/j.toxicon.2019.11.011

LOPES, A. B.; OLIVEIRA, A. A.; DIAS, F. C. F.; SANTANA, V. M. X.; OlIVEIRA, V. S.; LIBERATO, A. A.; GUEDES, V. R.. Perfil Epidemiológico Dos Acidentes Por Animais Peçonhentos $\mathrm{Na}$ Região Norte Entre Os Anos De 2012 E 2015. Revista de Patologia do Tocantins, v.4, n.2, p.36-40, 2017.

MARTINS, A.; JUNIOR, M. R. B.. Accidents with venomous animals of the order hymenoptera (bees and wasps): main complications in Latin American and Caribbean countries. Brazilian Journal of Health Review, v.1, n.1, p.220-232, 2018.

OLIVEIRA, S. S.; SOUSA, L. A. F.; ALVES, E. C.; FERREIRA, L. C. L.; SILVA, I. M.; LACERDA, M. V. G.; MONTEIRO, W. M.. Fatal stroke after Bothrops snakebite in the Amazonas state, Brazil: A case report. Toxicon, v.138, p.102-106, 2017.

OMS. Organização Mundial da Saúde. Doenças Tropicais Negligenciadas. Genebra: OMS, 2019.

PINTO, G. P. D. S.; ANDRADE, L. P. D.. Perfil Epidemiológico Dos Acidentes Com Animais Peçonhentos No Hospital 
Público Referência De Mato Grosso Do Sul. 2019.

SALVI, F. I.; PAULA, J. M.; LUTINSKI, J. A.; FRITZEN, D. M. M.; GIACHINI, K.; SCHABAT, F. M.; BUSATO, M. A.. Perfil Epidemiológico Dos Atendimentos Antirrábicos Humanos No Município De Chapecó, SC. Revista Interdisciplinar de Estudos em Saúde, v.7, n.1, p.176-186, 2018.

SILVA, A. M. D.; COLOMBINI, M.; SILVA, A. M. M.; SOUZA, R. M. D.; MONTEIRO, W. M.; BERNARDE, P. S.. Epidemiological and clinical aspects of snakebites in the upper Juruá River region, western Brazilian Amazônia. Acta Amazônica, v.50, n.1, 2019. DOI: https://doi.org/10.1590/18094392201901561

SILVA, C. F. R.; SANTOS, C. M.; PALERMO, T. A. C.. Perfil epidemiológico de acidentes por animais peçonhentos. Revista de Enfermagem da UFPI, v.7, n.3, p.35-41, 2018.

SILVA, M. J. C.; SOARES, F. T.; TRINDADE, G. P.; DINIZ, H. S.;
MEDEIROS, J. M. R.; LOPES, J. G. M.; NUNES, P. C..

Epidemiological profile of the ophidian accidents of lower Amazon region of Pará state, Brazil. Brazilian Journal of Health Review, v.2, n.3, p.1968-1979, 2019.

SOARES, F. G. S.; SACHETT, J. D. A. G.. Caracterização dos acidentes com animais peçonhentos: as particularidades do interior do Amazonas1. Scientia Amazônia, v.8, n.3, 2019

SOUZA, R. F.; NASCIMENTO, S. L.. Doenças e Agravos no Contexto das Grandes Inundações Graduais no Estado do Amazonas-Brasil. Hygeia, v.13, n.26, p.139-147, 2017.

VIEIRA, G. P. S.; MACHADO, C.. Accidents for venomous animals in the mountain region, Rio de Janeiro, Brazil. Journal Health Npeps, v.3, n.1, p.211-227, 2018.

VITÓRIA, E.. Perfil Epidemiológico De Pacientes Vítimas De Acidentes Por Animais Peçonhentos No Estado De Minas Gerais, Brasil: A Relação Entre Trabalho, Saúde E Ambiente. 2019.

A CBPC - Companhia Brasileira de Produção Científica (CNPJ: 11.221.422/0001-03) detém os direitos materiais desta publicação. Os direitos referem-se à publicação do trabalho em qualquer parte do mundo, incluindo os direitos às renovações, expansões e disseminações da contribuição, bem como outros direitos subsidiários. Todos os trabalhos publicados eletronicamente poderão posteriormente ser publicados em coletâneas impressas sob coordenação da Sustenere Publishing, da Companhia Brasileira de Produção Científica e seus parceiros autorizados. Os (as) autores (as) preservam os direitos autorais, mas não têm permissão para a publicação da contribuição em outro meio, impresso ou digital, em português ou em tradução. 